\title{
Telehealth Decreases Rural Emergency Department Wait Times for Behavioral Health Patients in a Group of Critical Access Hospitals
}

Roseanne Moody Fairchild, PhD, RN, CNE, NE-BC, ${ }^{1}$

Shiaw-Fen Ferng-Kuo, PhD, MPH, ${ }^{2}$ Stephanie Laws, MSN, $R N_{1}{ }^{3}$

Hicham Rahmouni, MBA, and Daniel Hardesty, ${ }^{3}{ }^{3} A^{3}$

${ }^{1}$ Nursing and Health Professions, State College of Florida, Manatee-Sarasota, Sarasota, Florida.

${ }^{2}$ Department of Applied Health Sciences, Indiana State University, Terre Haute, Indiana.

${ }^{3}$ Union Hospital, Richard Lugar Center for Rural Health, Terre Haute, Indiana.

\section{Abstract}

Background: Telehealth has been proposed as an important care delivery strategy to increase access to behavioral health care, especially in rural and medically-underserved settings where mental health care provider shortage areas predominate, to speed access to behavioral health care, and reduce health disparities. Introduction: This study was conducted to determine the effects of telehealth-based care delivery on clinical, temporal, and cost outcomes for behavioral health patients in rural emergency departments (EDs) of four Midwestern critical access hospitals (CAHs).

Materials and Methods: Observational matched cohort study of adult (age $\geq 18$ years) behavioral health patients treated in participating CAH EDs from 2015 to $2017(N=287)$. Telehealth cases were matched 2:1 retrospectively to nontelehealth control cases based on gender, age \pm 10 years, diagnosis group, and $C A H$, before implementation of telehealth in the rural hospitals (2005-2013; $N=153)$.

Results: The greatest number of behavioral health cases evaluated was in the mood, anxiety, and other mental health disorders category. The majority of patients in the telehealth (74\%) and nontelehealth (68\%) cohorts were 18-44 years. Mean ED wait time for the telehealth cohort was significantly shorter at 12 min (95\% CI 11-14 min) $(p<0.001)$ compared to a mean time of $27 \mathrm{~min}$ (95\% CI 22-32 $\mathrm{min}$ ) for the nontelehealth case controls (local provider only). The ED length of stay (LOS) for the telehealth cohort was significantly longer $(M=318 \mathrm{~min} v \mathrm{~s}$. $147 \mathrm{~min}, p<0.001)$ compared to the nontelehealth cohort. The end of telehealth visit to departure (EOTVtD) from the ED in minutes was evaluated to highlight factors potentially influencing delivery of behavioral health care in the ED. Across three behavioral diagnostic categories, time in minutes from end of telehealth visit to disposition/discharge was significantly longer for suicide and intentional self-injury cases $(n=100 ; 113 \mathrm{~min}$, 95\% CI 88-145; $p=0.004)$ compared to anxiety, mood, and other mental health disorders ( $n=126 ; 66 \mathrm{~min}, 95 \%$ CI 52-83). There was a clinically meaningful difference in EOTVtD in minutes for substance abuse-related cases, which were shorter in length on average ( $n=58 ; 71 \mathrm{~min}, 95 \%$ CI 54-94). Total ED costs for substance abuse-related cases for the telehealth ( $n=58$; \$4556, 95\% CI \$3963-\$5238) cohort were significantly higher than for the two other behavioral diagnostic groups $(p<0.001)$. Conclusions: Telehealth consultation in the ED for behavioral health cases was associated with decreased wait time and longer ED LOS. Similar to recent studies, the most common behavioral health cases involved mood and anxiety disorders. Costs related to treatment were highest for substance abuse-related cases, likely due to the additional interventions needed, especially related to resuscitation There are opportunities to improve ED efficiencies and post-telehealth visit protocols related to the timeframe extending from the EOTVtD from the $E D$, which continues to be a focus of future research. Additional research is also needed to determine if telehealth lends itself more effectively to specific categories of behavioral health cases.

Keywords: telehealth, emergency department, telebehavioral health, critical access hospital, rural health, length of stay, wait time, time-to-provider, telemedicine

\section{Introduction}

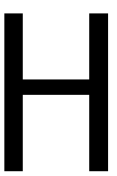

ealth care organizations across the United States (U.S.) continue to work to achieve the goals of the Institute for Health care Improvement's Triple Aim: (1) improving patient care experience, (2) enhancing population health, and (3) reducing costs of health care. ${ }^{1}$ Delivery of health care through telehealth typically involves a real-time, secure, internet-based care visit with a licensed provider who can improve access to targeted health assessment and intervention. ${ }^{1,2}$ While implementation models for various applications of telehealth are currently being tested, this virtual mode of care delivery is often highlighted as a 


\section{TELEBEHAVIORAL HEALTH IN RURAL EMERGENCY DEPARTMENTS}

valuable tool to improve speed to care, increase access to specialty care, and reduce health disparities in rural and urban medically underserved areas. ${ }^{1-6}$

Telehealth is increasingly viewed as an effective way to deliver psychiatric consultations across the lifespan to promote population health, and to provide ready access to patient-centered psychiatric care management for those with mental illness. ${ }^{4,5}$ As experts point out, implementation of telehealth in U.S. health care systems can improve behavioral population management by helping provider teams: (1) “...overcome geographic barriers to psychiatric care," (2) "...reduce rural health disparities in mental health treatment," and (3)"...improve the capacity for care by using synchronous and asynchronous consultation...to improve the skill level of

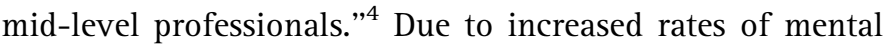
illness and the fragmentation of mental health resources across U.S. communities, ${ }^{4}$ the number of patients requiring behavioral health treatment who present to U.S. emergency departments (EDs) is on the rise. ${ }^{7,8}$ Rates of ED visits increased from $\sim 14.1$ ED visits to 20.3 ED visits per 1,000 population during 2006$2014 .^{8}$ During this same time period, the rate of mental health and substance abuse-related ED visits increased $\sim 44 \%{ }^{8}$

Approximately $96 \%$ of U.S counties have a shortage of psychiatrists to treat people with mental illness. ${ }^{8}$ Thus, telehealth-based behavioral health care is becoming a widely accepted mechanism to help address mental health care provider shortages in many rural and urban mental health care provider shortage areas (HPSAs). ${ }^{2,7,8}$ Therefore, behavioral health care delivery through telehealth has been viewed as “.... a tenable solution for addressing the shortage of psychiatric consultation to EDs in light of increasing demand for mental health treatment in the ED." ${ }^{2}$ Since rural U.S. counties often do not have any psychiatric or behavioral counseling care providers practicing within their county, ${ }^{9-11}$ attention must be paid to supporting increased access to specialty care in behavioral health for rural settings.

Experts have suggested that telehealth is likely to improve care in rural settings by promoting and sustaining the education of rural care providers in the targeted health conditions for which telehealth is implemented. ${ }^{4,6}$ The collaborative provider practice that is inherent in telehealth care delivery may create a "learning effect" ${ }^{\prime 6}$ "case-based learning", that fosters enhanced clinical knowledge regarding differential diagnosis and treatment for different behavioral health conditions among rural provider teams. Such effects would also inform ED providers regarding which types of behavioral health diagnoses are likely to benefit the most from a telebehavioral specialty visit immediately, compared to which behavioral health cases are first and foremost a medical air- way, breathing, and circulation emergency. This important distinction prompts near-immediate activation (or not) of a telehealth visit, based on the initial attending provider's assessment of the presenting patient.

Mohr et al., ${ }^{6,12}$ have reported that telehealth can create efficiencies in the ED regarding timeliness of care and length of stay (LOS) for trauma and other physical health conditions. Their recent efforts have revealed that access to telehealth can benefit rural ED patients who have been severely injured, ${ }^{6}$ and can expedite transfer of rural ED patients who need it. ${ }^{12}$ The authors have suggested that more study is needed to discern the impact of the availability of telehealth on different types of clinical ED care, as well as which patients are most likely to benefit from telehealth. ${ }^{6,12}$

As the application of telehealth in the delivery of behavioral health care is increasing, updated professional guidance regarding behavioral care delivery through telehealth has recently been released; this professional collaborative group recommends that effective psychiatric or behavioral health care can be delivered solely through telehealth. ${ }^{13}$ Several researchers have also reported that, at a minimum, telebehavioral care is noninferior to in-person behavioral care, ${ }^{14-16}$ and patients with some behavioral health conditions may even experience improved health outcomes through telebehavioral care compared to in-person care. ${ }^{17}$ Access to telebehavioral care has also been reported to reduce the need for inpatient hospitalization. ${ }^{5}$ More research is needed regarding temporal, disposition, and cost outcomes for patients with behavioral health diagnoses, who are treated with telehealth in rural EDs to explore any differences in these ED patient outcomes, ${ }^{6,12}$ based on different behavioral health diagnoses. ${ }^{4,5,18}$

The objective of this study was to evaluate the impact of a rural Indiana telehealth network delivering behavioral health consultations through a live, video-based interactive telehealth network in the EDs of four critical access hospitals (CAHs) over a period of 3 years. The study team evaluated cohort differences in the outcome variables, including: (1) ED wait time (time-to-provider), (2) ED LOS, (3) patient transfer status, (4) disposition of patient, and (5) primary payor. In addition, analyses of total cost, time, and disposition variables were conducted across major behavioral health diagnosis groups for telehealth cases, in an attempt to describe any potential gap or difference in patient work flow based on behavioral health diagnosis group.

\section{Materials and Methods}

STUDY DESIGN, SETTING, AND POPULATION

This study utilized an observational matched cohort design to evaluate outcomes for adult (age $\geq 18$ years) behavioral 


\section{FAIRCHILD ET AL.}

health patients who presented to the rural EDs between January 2015 and December 2017 and who received telehealthbased care by the Wabash Valley Rural Telehealth Network (WVRTN). The telehealth cohort cases were evaluated across the aforementioned study endpoints in comparison to retrospectively matched cases for behavioral health patients who were cared for in the participating rural EDs before the implementation of telehealth. The case controls originated during 20052013, depending on when the rural CAH adopted telehealth in their ED and the availability of the match based on the matching criteria (discussed in the Data Management section below).

The WVRTN serves some of the state's most economically disadvantaged and medically underserved counties in Indiana (Greene, Parke, Putnam, Sullivan, Vermillion, and Vigo), including a predominance of county-level federal HPSAs in mental health care, and a disproportionate number of individuals and families living at or below the poverty level. ${ }^{9-11}$ Combined economic data for the targeted region reveal that $10.4 \%$ of the population are living under $100 \%$ of the federal poverty level (FPL), and $30.18 \%$ of the population are living at or below $200 \%$ of the FPL. ${ }^{9-11}$

This study was approved by the regional university's Institutional Review Board. Data and outcomes are reported based on guidelines established by Strengthening the Reporting of Observational studies in Epidemiology (STROBE) statement. ${ }^{19}$ The project was funded by the Health Resources and Services Administration, Grant \# G01RH27871.

\section{STUDY PROTOCOL}

The WVRTN was the telehealth provider in the designated region during the study period. This rural regional network utilizes an on-demand "hub and spoke" design to deliver telehealth. The distant board-certified psychiatric providers comprise the central "hub" that connects to the rural ED CAH provider team (spoke), led by the board-certified emergency physicians in the rural ED as the attending providers. For this study, the telehealth visit was ordered by the attending physician, and was initiated by either the physician or by the registered nurse (RN) who had consulted with the attending ED physician.

Telebehavioral visits consisted of therapeutic patient interviews utilizing live video interaction in a private ED examination room with the distant psychiatrist, as initiated by the RN or physician, with one or both functioning in a facilitative role as needed. Once the telehealth-based visit was completed, the RN supported clinical documentation, electronic order entry, and patient disposition, including admission to the CAH if applicable, and transfers, or discharges from the CAH as necessary. Development and communication of a tailored behavioral health safety plan to the patient and sig- nificant other(s) relevant to patient disposition were also the responsibility of the $\mathrm{RN}$, in coordination with the distant psychiatrist and the attending ED physician.

The evaluation and outcomes of the participating rural ED health care provider teams have been reported elsewhere in relationship to perceptions of (1) their competency level, (2) the study's telehealth equipment, and (3) the implementation process in the participating rural EDs. ${ }^{20}$ Initial feedback provided by the ED health care provider teams during 2015 included the following: (1) poor internet connectivity and (2) cumbersome equipment that was too prone to breakage for telebehavioral visits.

Akin to an adaptive clinical study design, these issues were addressed during January-March 2016 as a result of this enduser feedback. During this time, the study team upgraded ethernet wireless connectivity in each CAH ED. In addition, instead of utilizing freestanding computers-on-wheels ("COWs") for telebehavioral visits, the study team provided military-grade hand-held tablets with a secure, encrypted platform that was HIPAA compliant for transmission of virtual telehealth visits, to streamline telebehavioral care delivery and guard against equipment breakage by agitated patient(s).

\section{KEY OUTCOME MEASURES}

The primary study endpoint was ED wait time, or time-toprovider, defined as the time interval in minutes from ED registration at the triage desk to contact by a licensed health care provider in the ED examination room (either a local provider or a telehealth provider). The research team hypothesized that ED wait time would be decreased due to the ready availability of a specialist psychiatric provider through telehealth. Thus, speed-to-care should be enabled through telehealth, since there were no specialty psychiatric providers working in the participating rural EDs during this study, and rural generalist ED care providers have reported a need for evidence-based support in assessing and treating different types of psychiatric conditions. ${ }^{2,4,5}$

Secondary endpoints explored operational metric variables describing ED "throughput," or patient flow through ED, for telehealth and nontelehealth cohorts. These endpoints included ED LOS (time from ED registration to discharge, transfer, or admission, in minutes), length of telehealth visit, and disposition of patient (admission to CAH, transfer to another facility, or discharge). Additional secondary endpoints included primary payor, and total cost of ED care (telehealth patients only), to provide context for socioeconomic status of patients. Primary payor categories to be reported were Medicaid, Medicare, private insurance, and self-pay/uninsured. Costs of care were reported as all costs of care incurred in the ED 
and which were ultimately billed per case for the telehealth cohort. All cost data were adjusted for inflation utilizing the Consumer Price Index, expressed in 2016 dollars per CPI. ${ }^{21,22}$

It is important to note that total ED costs are presented here for telehealth cases only due to two historically based confounding variables. First, telehealth cases will logically add an additional cost in the form of a charge for ED care simply due to the addition of this specialty-driven consultation that was not available pre-telehealth implementation. A second confounding variable was that the Centers for Medicare and Medicaid (CMS) began to enforce meaningful use practices with hospitals through adjustments to Medicare and Medicaid reimbursements for hospital noncompliance beginning in 2015. This change impacted hospital charges to patients during the compliance efforts timeframe after 2015, during which timeframe the cost data for the telehealth cohort were collected. ${ }^{23}$

The descriptive study endpoints selected for analysis align with the research team's active involvement in the establishment of a standardized set of outcome measures for telehealth studies through a Health Resource and Services Administration (HRSA)-sponsored 2015 pilot study. ${ }^{24}$ In addition, an exploratory endpoint evaluated for telehealth cases only, was the end of telehealth visit to departure (EOTVtD) from the ED (minutes), which was not a part of the original standardized set of teleemergency outcome measures proposed. ${ }^{24}$ This exploratory endpoint may shed light on contextual issues of care delivery for behavioral health in particular. Observing the length of portions of the ED cohort visits allowed for assessment of any differences related to study variables and timeliness of care.

\section{DATA MANAGEMENT}

Patient diagnoses for the telehealth cases were identified and matched 2:1 to nontelehealth patient cases that had been treated in the ED before implementation of telehealth in the participating hospitals (2005-2013; $N=153)$. Matches were based on primary diagnosis code for each behavioral health case, as well as gender, age \pm 10 years, and CAH (see Table 1). A 2:1 rather than a 1:1 matching process was implemented due to the level of availability of appropriately matched nontelehealth cases based on the aforementioned matching criteria. Records were deidentified internally and then retrieved onsite from each participating CAH's medical records department through paper medical records or electronic health records. Diagnosis codes were then translated from International Classification of Diseases, 9th edition (ICD-9) to the new ICD-10 version, as this switch occurred in the participating CAHs during the second year (2016) of the study.

After the translation from ICD-9 to ICD-10 coding, all behavioral cases were subsequently grouped based on behav- ioral health diagnostic categories for statistical analysis purposes. For example, suicide, suicide attempt, suicidal ideation, and self-inflicted injury were grouped together as the broad category of suicide and intentional self-inflicted injury for statistical evaluation. Table 2 presents the broad behavioral health category groups evaluated for the study based on the Clinical Classification Software categorization available from the Agency for Health care Research and Quality. ${ }^{25}$

\section{DATA ANALYSIS}

This study involved an observational matched cohort evaluation of adult (age $\geq 18$ years) behavioral health patients

Table 1. Demographic Characteristics of Matched Cases

TELEHEALTH NONTELEHEALTH

$N(\%) \quad N(\%)$

\begin{tabular}{|c|c|c|}
\hline \multicolumn{3}{|l|}{ Age } \\
\hline $18-24$ & $96(33 \%)$ & $42(28 \%)$ \\
\hline $25-44$ & $118(41 \%)$ & $61(40 \%)$ \\
\hline $45-64$ & $64(22 \%)$ & $42(28 \%)$ \\
\hline $65-74$ & $6(2 \%)$ & $4(3 \%)$ \\
\hline $75+$ & $3(1 \%)$ & $4(3 \%)$ \\
\hline Total & 287 & 153 \\
\hline \multicolumn{3}{|l|}{ Gender } \\
\hline Female & $121(42 \%)$ & $66(43 \%)$ \\
\hline Male & $166(58 \%)$ & 87 (57\%) \\
\hline Total & 287 & 153 \\
\hline \multicolumn{3}{|l|}{ Principal diagnosis categories } \\
\hline $\begin{array}{l}\text { Anxiety, mood, and other mental } \\
\text { health disorders }\end{array}$ & $126(44 \%)$ & $92(60 \%)$ \\
\hline $\begin{array}{l}\text { Suicide and intentional } \\
\text { self-inflicted injury }\end{array}$ & $102(36 \%)$ & $34(22 \%)$ \\
\hline Substance abuse related & $59(21 \%)$ & $27(18 \%)$ \\
\hline Total & 287 & 153 \\
\hline \multicolumn{3}{|l|}{ Payors } \\
\hline Medicaid only & $125(52 \%)$ & $24(20 \%)$ \\
\hline Medicare only & $18(8 \%)$ & 19 (15\%) \\
\hline Medicaid/medicare & $8(3 \%)$ & $3(2 \%)$ \\
\hline Private & $49(20 \%)$ & $42(34 \%)$ \\
\hline Self-pay/uninsured & $40(17 \%)$ & $35(28 \%)$ \\
\hline Total & 240 & 123 \\
\hline
\end{tabular}




\section{FAIRCHILD ET AL.}

Table 2. Categorization of Patient Diagnosis Codes

\section{for Statistical Analysis}

Suicide and intentional Suicide, suicide attempt, suicidal ideation, and self-inflicted injury

Substance abuse related Substance use/substance abuse, alcohol intoxication, overdose, and accidental poisoning

Anxiety, mood, and other Anxiety disorders, mood (depression and bipolar) mental health disorders disorders, personality, post-traumatic stress, and adjustment disorders

treated in participating CAH EDs from 2015 to $2017(N=287)$. Among the total number of patients considered for telehealthbased care in participating rural EDs $(N=522), 235$ patients were subsequently triaged to neurological care diagnoses, which will be evaluated in a separate work. Thus, for data analysis purposes, the research team examined study variable relationships using a matched 2:1 case-control approach for telebehavioral cases [ $n=287]$ compared to nontelehealth behavioral cases $[n=153])$. Data were analyzed using the IBM SPSS $^{\circledR}$ version 25.0 (IBM SPSS Statistics for Windows, Armonk, NY) and statistical significance was defined as $\alpha<0.05$.

Variable relationships were evaluated utilizing descriptive, nonparametric, and parametric statistical tests. The parametric statistical analysis included (1) Student's $t$ test (twotailed) to determine any significant difference in outcomes between telehealth and nontelehealth behavioral health cases and (2) analysis of variance (ANOVA) to determine any significant difference across the three broad categories of behavioral health diagnoses (suicide, substance abuse, and anxiety, mood, and other mental health disorders) in relation to ED throughput metrics, patient disposition, total cost, and primary payor. The timeliness, disposition, and telehealth cohort cost data were positively skewed rather than normally distributed as per the Kolmogorov-Smirnov test; thus, these data were log-transformed for parametric analysis purposes. Anti-log (back transformed) values for timeliness, disposition, and cost data are presented for ease of interpretation and reporting purposes (Tables 3 and 4). ${ }^{26,27}$

The nonparametric statistical analysis involved chi-square tests to determine any potential relationship between the following: (1) behavioral patient disposition for telehealth and nontelehealth cohorts; (2) age range and category of behavioral health diagnosis; and (3) primary payor for telehealth and nontelehealth cohorts. Cases with missing data related to variables of interest were not included in the analyses.

\section{Results}

Demographics for all cases in the matched telehealth and nontelehealth case cohorts are described in Table 1. The racial ethnicity for both cohorts was $\sim 98.5 \%$ White, which was representative of the general population in the participating counties, ${ }^{9}$ with similar distributions across gender and age range categories. Payor mix, however, was different for telehealth compared to nontelehealth cases. For prospective telehealth cases, the number attributed to Medicaid was 32\% higher than for nontelehealth cases. Using chi-square analysis, there was a significantly greater number of Medicaid patients in the telehealth cohort $(n=125 ; 52 \% ; p<0.001)$ compared to nontelehealth cohort ( $n=24 ; 20 \%$ ) (Table 1). The greatest number of behavioral health cases evaluated were in the mood, anxiety, and other mental health disorders category (Table 1), and the majority of patients in the telehealth (74\%) and nontelehealth (68\%) cohorts were 18-44 years of age.

\section{PRIMARY OUTCOME: TIME-TO-PROVIDER}

The research team hypothesized that ED wait time would be decreased due to the ready availability of a specialist psychiatric provider through telehealth. Mean wait time, or mean time in minutes until the patient was seen by a provider in the

Table 3. Emergency Department Wait Time (Time-to-Provider) (Minutes) for Telehealth and Nontelehealth Emergency Department Visits

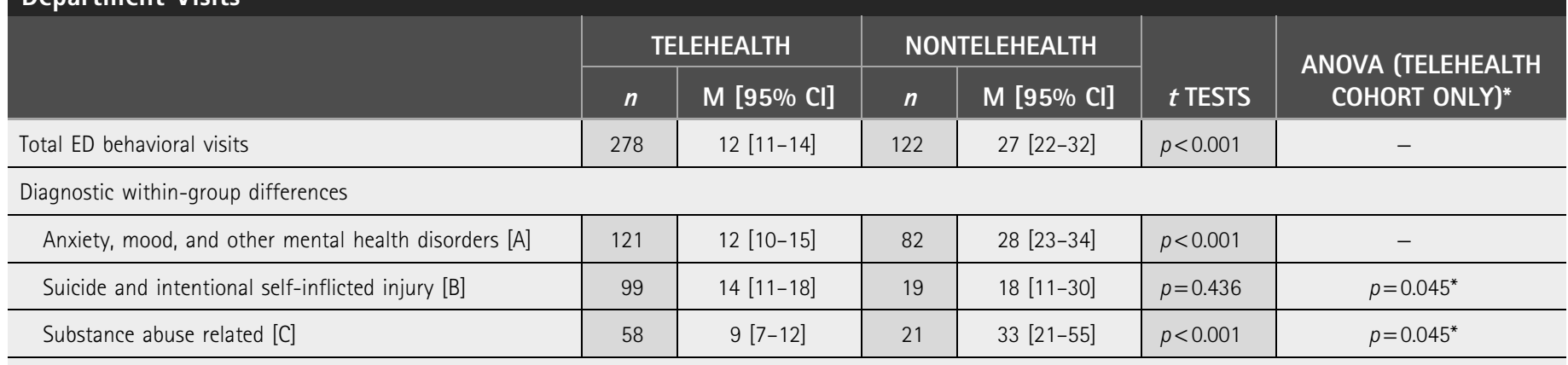

${ }^{*}$ Bonferroni post hoc test $[\mathrm{B}]:[\mathrm{C}]: p=0.049$ 


\begin{tabular}{|c|c|c|c|c|c|c|}
\hline & & \multicolumn{2}{|c|}{ TELEHEALTH } & \multicolumn{2}{|c|}{ NONTELEHEALTH } & \multirow[b]{2}{*}{$\mathrm{t}$ TESTS } \\
\hline & & $n$ & $\mathrm{M}[95 \% \mathrm{Cl}]$ & $n$ & $\mathrm{M}[95 \% \mathrm{Cl}]$ & \\
\hline \multirow[t]{5}{*}{ ED wait time (time-to-provider), min } & Total behavioral ED visits & 249 & $12[11-14]$ & 117 & 27 [23-33] & $p<0.001$ \\
\hline & Patient disposition: & & & & & \\
\hline & Admitted to $\mathrm{CAH}[\mathrm{A}]$ & 38 & 13 [9-20] & 28 & $27[18-40]$ & $p=0.019$ \\
\hline & Routine discharge [B] & 133 & $12[10-15]$ & 61 & $28[22-36]$ & $p<0.001$ \\
\hline & Transfer [C] & 78 & $12[9-15]$ & 28 & 26 [16-41] & $p=0.004$ \\
\hline \multicolumn{2}{|c|}{ Across dispositions: ANOVA: } & \multicolumn{2}{|c|}{$p=0.198$ Nonsignificant } & \multicolumn{2}{|c|}{$p=0.929$ Nonsignificant } & \\
\hline \multirow[t]{5}{*}{ ED Total Length of Stay (LOS), min } & Total behavioral ED visits & 256 & 318 [298-338] & 124 & 147 [130-167] & $p<0.001$ \\
\hline & Patient disposition: & & & & & \\
\hline & Admitted to $\mathrm{CAH}[\mathrm{A}]$ & 41 & 332 [292-378] & 28 & 186 [150-229] & $p<0.001$ \\
\hline & Routine discharge [B] & 136 & $255[236-276]$ & 62 & $107[90-126]$ & $p<0.001$ \\
\hline & Transfer [C] & 79 & $452[410-498]$ & 34 & $218[173-274]$ & $p<0.001$ \\
\hline \multicolumn{2}{|c|}{$\begin{array}{l}\text { Across dispositions: ANOVA: } \\
{ }^{*} \text { Bonferroni post hoc tests: }\end{array}$} & \multicolumn{2}{|c|}{$\begin{array}{c}p<0.001 \\
p=0.003[\mathrm{~A}]:[\mathrm{B}]^{*} \\
p<0.001[\mathrm{~A}]:[\mathrm{C}]^{*} \\
p=0.003[\mathrm{~B}]:[\mathrm{C}]^{*}\end{array}$} & \multicolumn{2}{|c|}{$\begin{array}{c}p<0.001 \\
p=.017[\mathrm{~B}]:[\mathrm{A}]^{*} \\
p<.001\left[\mathrm{~B}\left[:[\mathrm{C}]^{*}\right.\right.\end{array}$} & \\
\hline \multirow[t]{5}{*}{ Length of Telehealth Visit, min } & Total behavioral ED visits & \multicolumn{2}{|l|}{256} & \multicolumn{2}{|c|}{$55[52-57]$} & $\mathrm{n} / \mathrm{a}$ \\
\hline & Patient disposition: & & & & & \\
\hline & Admitted to $\mathrm{CAH}[\mathrm{A}]$ & \multicolumn{2}{|l|}{41} & \multicolumn{2}{|c|}{$57[52-63]$} & $\mathrm{n} / \mathrm{a}$ \\
\hline & Routine discharge [B] & \multicolumn{2}{|l|}{136} & \multicolumn{2}{|c|}{$54[51-57]$} & $\mathrm{n} / \mathrm{a}$ \\
\hline & Transfer [C] & \multicolumn{2}{|l|}{79} & \multicolumn{2}{|c|}{$54[50-59]$} & $\mathrm{n} / \mathrm{a}$ \\
\hline \multicolumn{2}{|c|}{ Across dispositions: ANOVA: } & \multicolumn{2}{|c|}{$p=0.660$ Nonsignificant } & \multicolumn{2}{|c|}{$\mathrm{n} / \mathrm{a}$} & \\
\hline
\end{tabular}

ANOVA, analysis of variance; $\mathrm{CAH}$, critical access hospitals; $\mathrm{Cl}$, confidence interval; ED, emergency department.

rural EDs for the telehealth cohort, was significantly shorter at $12 \mathrm{~min}$ (95\% CI 11-14 $\mathrm{min} ; p=0.012$ ) compared to nontelehealth case controls (local provider) ( $\mathrm{M}=27 \mathrm{~min}, 95 \% \mathrm{CI}$ 22-32 min) (Table 3). Time-to-provider was shortest for substance abuse-related cases.

\section{SECONDARY OUTCOMES}

ED length of stay. The ED LOS for telehealth cohort patients was significantly longer $(M=318 \mathrm{~min}$ vs. $M=147 \mathrm{~min}$, $p<0.001)$ compared to the nontelehealth cohort LOS; Tables 4 and 5 show stratified results. The overall LOS for behavioral patients receiving telehealth was $\sim 171$ min longer. When factoring in the mean length of the telehealth visit across all dispositions ( $M=57 \mathrm{~min}$; 95\% CI 55-59 min), there are $\sim 100$ min on average remaining in the ED visit for the telehealth cohort. Based on disposition status, patients who received telehealth had significantly longer ED LOS $(p<0.001)$ whether they were subsequently admitted to the $\mathrm{CAH}$, or discharged, or transferred to another facility.

Patient disposition. In comparing telehealth and nontelehealth cases in regard to patient disposition, a slightly larger, but nonsignificant proportion of patients were transferred to another facility in telehealth cohort (31\%) compared to nontelehealth (24\%). In addition, a smaller proportion of telehealth patients $(16 \%)$ were admitted to the CAH compared to nontelehealth (24\%). A similar proportion of telehealth (53\%) and nontelehealth (52\%) behavioral patients were routinely discharged; however, there was no significant difference in the disposition of ED behavioral health patients between the telehealth $(n=256)$ and nontelehealth $(n=125)$ cohorts ( $p=0.313)$.

Total cost of telehealth cases in the ED. Total ED costs for telehealth cohort $(\$ 3,649,95 \%$ CI $\$ 3,470-\$ 3,837 ; n=287)$ have been stratified and are presented across the 3 behavioral 


\begin{tabular}{|c|c|c|c|c|c|c|}
\hline & & \multicolumn{2}{|c|}{ TELEHEALTH } & \multicolumn{2}{|c|}{ NONTELEHEALTH } & \multirow[b]{2}{*}{ t TESTS } \\
\hline & & $n$ & $\mathrm{M}[95 \% \mathrm{Cl}]$ & $n$ & $\mathrm{M}[95 \% \mathrm{Cl}]$ & \\
\hline \multirow[t]{6}{*}{ ED wait time (time-to-provider), min } & Total behavioral ED visits & 278 & $12[11-14]$ & 122 & $27[22-32]$ & $p<0.001$ \\
\hline & Diagnostic within-group differences & & & & & \\
\hline & Anxiety, mood, and other mental health disorders $[A]$ & 121 & $12[10-15]$ & 82 & $28[23-34]$ & $p<0.001$ \\
\hline & Suicide and intentional self-inflicted injury [B] & 99 & $14[11-18]$ & 19 & $18[11-30]$ & $p=0.436$ \\
\hline & Substance abuse related $[\mathrm{C}]$ & 58 & $9[7-12]$ & 21 & $33[21-55]$ & $p<0.001$ \\
\hline & Across diagnostic groups: ANOVA: *Bonferroni post hoc tests & \multicolumn{2}{|c|}{$\begin{array}{c}p=0.045 \\
p=0.049[B]:[C]^{*}\end{array}$} & \multicolumn{2}{|c|}{$\begin{array}{c}p=0.125 \\
\text { Nonsignificant }\end{array}$} & - \\
\hline \multirow[t]{6}{*}{ ED total length of stay (LOS), min } & Total behavioral ED visits & 287 & $324[305-343]$ & 129 & $148[130-168]$ & $p<0.001$ \\
\hline & Diagnostic within-group differences & & & & & \\
\hline & Anxiety, mood, and other mental health disorders $[A]$ & 126 & $303[278-331]$ & 82 & $134[113-158]$ & $p<0.001$ \\
\hline & Suicide and intentional self-inflicted injury [B] & 102 & $368[332-407]$ & 25 & $169[120-237]$ & $p<0.001$ \\
\hline & Substance abuse related $[\mathrm{C}]$ & 59 & $297[264-335]$ & 22 & $183[146-230]$ & $p<0.001$ \\
\hline & Across diagnostic groups: ANOVA: *Bonferroni post hoc tests & \multicolumn{2}{|c|}{$\begin{array}{c}p=0.005 \\
p=0.011[\mathrm{~A}]:[\mathrm{B}]^{*} \\
p=0.027[\mathrm{~B}]:[\mathrm{C}]^{*}\end{array}$} & \multicolumn{2}{|c|}{$\begin{array}{c}p=0.122 \\
\text { Nonsignificant }\end{array}$} & - \\
\hline \multirow[t]{6}{*}{ Length of telehealth visit, min } & Total behavioral ED visits & \multicolumn{2}{|l|}{287} & \multicolumn{2}{|r|}{$55[53-57]$} & $\mathrm{n} / \mathrm{a}$ \\
\hline & Diagnostic within-group differences & & & & & \\
\hline & Anxiety, mood, and other mental health disorders $[A]$ & \multicolumn{2}{|l|}{126} & \multicolumn{2}{|c|}{$54[51-58]$} & $\mathrm{n} / \mathrm{a}$ \\
\hline & Suicide and intentional self-inflicted injury [B] & \multicolumn{2}{|l|}{102} & \multicolumn{2}{|c|}{$57[53-61]$} & $\mathrm{n} / \mathrm{a}$ \\
\hline & Substance abuse related $[\mathrm{C}]$ & \multicolumn{2}{|l|}{59} & \multicolumn{2}{|c|}{$52[48-57]$} & $n / a$ \\
\hline & Across diagnostic groups: ANOVA & \multicolumn{4}{|c|}{$\begin{array}{c}p=0.299 \\
\text { Nonsignificant }\end{array}$} & $\mathrm{n} / \mathrm{a}$ \\
\hline
\end{tabular}

ANOVA, analysis of variance; $\mathrm{Cl}$, confidence interval; ED, emergency department.

\begin{tabular}{|c|c|c|c|c|c|}
\hline & $\begin{array}{l}\text { ADMITTED TO CAH } \\
\text { M [95\%Cl] [A] }\end{array}$ & $\begin{array}{c}\text { ROUTINE DISCHARGE } \\
\text { M }[95 \% \mathrm{Cl}][\mathrm{B}]\end{array}$ & $\begin{array}{c}\text { TRANSFER } \\
\mathrm{M}[95 \% \mathrm{Cl}][\mathrm{C}]\end{array}$ & ANOVA $^{a}$ & $p$ VALUE $^{b}$ \\
\hline PATIENT DISPOSITION & $n=41$ & $n=136$ & $n=79$ & & \\
\hline $\begin{array}{l}\text { End of Telehealth Visit to Departure } \\
\text { from the ED (EOTVtD), min }\end{array}$ & 127 [98-163] & $37[30-44]$ & 181 [144-228] & $p<0.001$ & $\begin{array}{l}p<0.001[\mathrm{~A}]:[\mathrm{B}] \\
p<0.001[\mathrm{~B}]:[\mathrm{C}]\end{array}$ \\
\hline Total Cost of ED Stay & $6,261[5,455-7,186]$ & $3,160[2,971-3,361]$ & $3,450[3,192-3,729]$ & $p<0.001$ & $\begin{array}{l}p<0.001[A]:[B] \\
p<0.001[A]:[C]\end{array}$ \\
\hline
\end{tabular}

\begin{tabular}{|c|c|c|c|c|c|}
\hline $\begin{array}{c}\text { PATIENT DIAGNOSTIC } \\
\text { GROUP }\end{array}$ & $\begin{array}{c}\text { ANXIETY, MOOD, AND OTHER } \\
\text { MENTAL HEALTH DISORDERS } \\
\text { M }[95 \% \mathrm{Cl}][\mathrm{A}]\end{array}$ & $\begin{array}{l}\text { SUICIDE AND INTENTIONAL } \\
\text { SELF-INFLICTED INJURY } \\
\text { M [95\% Cl] [B] }\end{array}$ & $\begin{array}{c}\text { SUBSTANCE ABUSE } \\
\text { RELATED } \\
\mathrm{M}[95 \% \mathrm{Cl}][\mathrm{C}]\end{array}$ & ANOVA $^{a}$ & $p$ VALUE $^{b}$ \\
\hline & $n=126$ & $n=100$ & $n=58$ & - & - \\
\hline EOTVtD from the ED, min & 66 [52-83] & 113 [88-145] & $71[54-94]$ & $p<0.001$ & $p=0.004[A]:[B]$ \\
\hline Total cost of ED stay & $3,355[3,152-3,572]$ & $3,559[3,276-3,867]$ & $4,556[3,963-5,238]$ & $p<0.001$ & $\begin{array}{l}p<0.001[A]:[C] \\
p<0.001[B]:[C]\end{array}$ \\
\hline
\end{tabular}

aStatistical significance of ANOVA results based on disposition status or diagnostic group.

${ }^{b} p$ values for Bonferroni post hoc tests when ANOVA results are significant.

ANOVA, analysis of variance; $\mathrm{CAH}$, critical access hospitals; $\mathrm{Cl}$, confidence interval; ED, emergency department; EOTVtD, end of telehealth visit to departure. 


\section{TELEBEHAVIORAL HEALTH IN RURAL EMERGENCY DEPARTMENTS}

diagnosis groups (Table 6). Total ED costs for substance abuse-related cases for telehealth $(n=59 ; \$ 4,556,95 \% \mathrm{CI}$ $\$ 3,963-\$ 5,238$ ) cohort were significantly higher than the 2 other behavioral diagnostic groups $(p<0.001)$. There was no significant difference in total ED costs between anxiety, mood, and other mental health disorders and suicide and intentional self-inflicted injury for the telehealth cohort.

Exploratory telehealth endpoint. The EOTVtD from the ED in minutes was evaluated for telehealth cases only, to highlight possible factors influencing the delivery of behavioral care. Across the three behavioral diagnostic categories, time in minutes from the end of telehealth visit to disposition/departure was significantly longer for suicide and intentional self-injury cases ( $n=100 ; 113 \mathrm{~min}, 95 \%$ CI 88-145; $p=0.004$ ) compared to anxiety, mood, and other mental health disorders ( $n=126 ; 66 \mathrm{~min}, 95 \%$ CI 52-83). There was also a clinically meaningful difference in EOTVtD in minutes for substance abuse-related cases, which were also shorter in length $(n=58$; $71 \mathrm{~min}, 95 \%$ CI 54-94).

Based on patient disposition, EOTVtD was significantly shorter for telebehavioral patients who were routinely discharged ( $n=136 ; 37$ min, 95\% CI 30-44; $p<0.001$ ) compared to patients admitted to $\mathrm{CAH}(n=41 ; 127 \mathrm{~min}, 95 \% \mathrm{CI} 98-163)$ and patients who were transferred to another facility $(n=79$; $181 \mathrm{~min}, 95 \%$ CI 144-228). In addition, the EOTVtD for government payor patients (Medicaid and Medicare) $(n=172$; $86 \mathrm{~min}, 95 \%$ CI 71-103) was slightly longer than for nongovernment payor patients $(n=95 ; 71 \mathrm{~min}, 95 \%$ CI 55-92; $p=0.783)$. Contextual factors relating to complexities of treatment for behavioral health cases in rural settings are presented below for discussion and consideration in relationship to study results and areas for future research.

\section{Discussion}

Telehealth is currently being studied as a lever to improve operational metrics and throughput, or patient flow, in the ED, for different types of health conditions. ${ }^{6,12}$ The number of patients presenting to U.S. EDs continues to grow, ${ }^{7,8}$ and behavioral health patients, in particular, comprise an increasing number of ED cases that need to be evaluated based on best evidence. $^{2,4}$ To this end, mean ED wait time (or time-toprovider) in this study was significantly shorter for behavioral health patients treated utilizing telehealth than for the nontelehealth cohort. Mohr et al. ${ }^{12}$ recently reported a similar result in an observational study of rural hospital EDs implemented in a telemedicine network in the Midwest U.S. As rural hospital generalist providers have been reported to experience educational benefits related to targeted specialty support through telehealth, ${ }^{2,4,5}$ perhaps this learning effect helps to promote greater understanding of patient needs for different behavioral health conditions, and thus shorter wait times, for this study.

ED LOS in this study was longer for telehealth patients, similar to results of two other recent studies of telehealth implementation in rural EDs. ${ }^{6,12}$ The authors of this study, two of whom are experienced ED nurses, propose two practicebased reasons to be considered for this LOS phenomenon, in addition to the basic time element that telehealth adds as an additional intervention: (1) treatment time in EDs typically becomes extended if the behavioral health patient needs to be admitted, due to the lack of availability of psychiatric beds at tertiary facilities for transfer patients and (2) LOS is lengthened due to the time it takes to prepare a room and/or wait for an in-hospital bed to become available for the next admission. Moreover, some behavioral health facilities do not accept Medicaid patients, which was the most common payor for telehealth patients in this study. This payor-related factor prolongs the time needed to locate an appropriate inpatient bed in behavioral health, especially in rural areas where there are fewer inpatient behavioral care facilities in general. ${ }^{10,11}$

Extended wait times after the telehealth visit are reflected in the "end of telehealth visit to departure from the ED," or EOTVtD variable. The EOTVtD is a new outcome measure proposed for the recent team-based report in which the study team participated, to support development of a standardized set of outcome measures for telehealth care delivery. ${ }^{24}$ This new variable, as it has been defined, allows researchers to explore areas with the potential for improved efficiencies related to ED telehealth care delivery post-ED telehealth visit.

Fellow researchers have also called for further examination of telehealth-driven workflow related to the complexity underlying ED LOS measurement. ${ }^{12}$ For this study, the lack of availability of psychiatric beds for transfer patients resulted in a prolonged EOTVtD time of 3 h (181 min; 95\% CI 144-228) (Table 6). Since there was a significantly greater number of Medicaid patients in the telehealth cohort for this study $(p<0.001)$, this contextual factor underscores the importance of evaluating the EOTVtD timeframe. Wait time after a telehealth visit in the ED subsequently impacts patient throughput, and potentially impacts patients' behavioral health outcomes, due to prolonged LOS. ${ }^{28}$

The prolonged waits for psychiatric bed transfers in EDs across the U.S. have been decried as a phenomenon that needs immediate attention, according to a recent mental health report to the U.S. Congress: "We intend to promote evidencebased practices and strong community-based systems of care, and to end travesties such as unnecessary incarceration and "boarding" of people with acute psychiatric conditions in EDs 


\section{FAIRCHILD ET AL.}

for hours or days. We hope that coordinated federal efforts will enable our partners in states and communities to strengthen their efforts in support of these goals." ${ }^{28}$ Commissioned by the U.S. Department of Health and Human Services through 2022, the subsequent recommendations of the 2017 report of the Interdepartmental Serious Mental Illness Coordinating Committee (ISMICC) included the following: "Use data to improve quality of care and outcomes," "Use telehealth and other technologies to increase access to care," and "Maximize capacity of the behavioral health workforce." ${ }^{28}$ It would seem that telehealth-based interventions would support these primary goals of the ISMICC.

Relevant to minimizing ED LOS, telehealth care delivery has become an important driver to support U.S. EDs in effectively serving increasing numbers of behavioral health patients. The behavioral health patient may be able to experience a brief check-in time, receive needed specialty care through a telehealth visit, and then a routine discharge. If ED efficiencies in wait time and LOS are realized with behavioral health patients, this may prompt creation of ED "psychiatric tracks" akin to the ED "fast tracks" developed over the past 20 years to improve patient flow for those presenting to the ED with primary/urgent care needs. Unless all rural Federally Qualified Health Centers (FQHCs) are staffed (virtually or physically) with licensed psychiatric providers, or the Certified Community Behavioral Health Clinic (CCBHC) program is expanded, ${ }^{28}$ would it be feasible for rural EDs to support "micro-telehealth hubs" and provide a central location for delivery of inpatient $\mathrm{BH}$ triage, as well as outpatient behavioral telehealth visits for their respective service counties/areas?

Carolinas $^{5}$ and Mayo Clinic ${ }^{2}$ are two major health systems that have established and are engaged in ongoing evaluation of innovative telehealth-based ED programs that have targeted $\mathrm{BH}$ patients. These creative approaches are improving access to care. ${ }^{2,5}$ Both programs are testing different patient throughput models similar to this study's "hub and spoke" design. "Hub" refers to the centrally located regional hospital that provides triage for, and subsequent connection to, the psychiatric specialty provider requests by the outlying "spokes," namely the participating rural CAHs. Tertiary outpatient and inpatient psychiatric facilities are few in number among rural regions across the U.S. ${ }^{11}$ Thus, the increased incidence of routine discharge with a tailored safety plan that was observed in this study for ED telebehavioral patients helped to improve efficiencies in the management of participating patients with chronic mental illness.

The ED costs for the study were highest for substance abuserelated patients ( $\mathrm{M}=\$ 4,556,95 \% \mathrm{CI} 3,963-5,238 ; p<0.001)$ compared to the other diagnostic groups (Table 6). This sig- nificant difference in costs reflected delivery of any emergent, life-saving care needed, as well as follow-up on any remaining patient care needs that ultimately added to the number of ED procedures subsequently performed post-telehealth visit. Primary reasons for higher costs for substance abuse patients are that substance abuse and/or overdose patients can require intubation and resuscitation, initial investigation of type of substance ingested, gastric lavage, chemical and/or physical restraint, and/or complex and ongoing IV medication delivery, depending on the substance(s) ingested and presence of agitation and/or psychotic signs and symptoms. Many substance abuse patients also receive a period of post-treatment ED observation before transfer or discharge, to assess and treat delirium tremens and/or determine the need for a detoxification admission. Thus, implementation of multiple complex assessments and interventions was a primary reason for higher costs for substance abuse-related cases.

Efforts are ongoing to decrease costs of health care, especially in U.S. EDs. ${ }^{2,5,7,8}$ As telehealth networks like the WVRTN expand and serve additional rural counties in behavioral health, economies of scale may allow mitigation of pertelehealth-visit costs based on an increased number of visits provided by the contracted behavioral care specialists. Economic evaluations suggest that telehealth networks with a higher number of behavioral patient encounters across multiple sites tend to have lower costs as they serve rural populations, producing economic benefits over time. ${ }^{2,5,29}$

For the secondary exploratory cost variable, this study may contribute new knowledge regarding the comparative ED costs for different types of telebehavioral cases, and overall costs of telebehavioral ED patients in relation to patient disposition. An important caveat concerning costs of ED telehealth visits for $\mathrm{BH}$ patients is the relationship between telebehavioral care delivery in the ED and the EOTVtD timeframe. Telehealth appears to shorten initial ED wait time across several recent studies, thus improving ready access to specialty psychiatric care; however, unless the patient is discharged to home and/or to outpatient care, this positive outcome may be subsequently blunted by the historically challenging issues related to long wait times for psychiatric beds, for patients who are waiting for transfers out of rural hospitals. ${ }^{2,5,28}$ In any case, additional research is needed to further refine telehealth-driven outcome measures ${ }^{18,24}$ in support of a collaborative national network for telehealth research. In this way, larger, prospective studies may be pursued to ascertain the value of telehealth for "different care delivery models and use cases." 18

This study has several limitations; thus, results should be interpreted cautiously. Utilizing an observational design, 


\section{TELEBEHAVIORAL HEALTH IN RURAL EMERGENCY DEPARTMENTS}

matched data were collected from previous years as case controls, which create a historical confounding factor, even though the patient case data were matched on key characteristics such as diagnosis, age \pm 10 years, gender, and rural hospital, and all cost data were aligned based on 2016 U.S consumer price index. The study team collected data in a consistent manner at the 4 participating Midwestern rural hospitals, but results are not necessarily generalizable to all rural CAHs; this study was not conducted as a randomized, controlled clinical trial with a predetermined power analysis to support optimal sample size across all U. S. CAHs.

\section{Conclusion}

In conclusion, telehealth consultation in the rural EDs for behavioral health cases was associated with decreased wait time and longer ED LOS, with higher relative costs reflected for substance abuse cases. However, an increased incidence of routine discharge for telebehavioral patients with a tailored safety plan was observed, which should improve efficiencies in the management of rural patients with chronic mental illness. There are opportunities to improve ED efficiencies related to the timeframe extending from the EOTVtD from ED. Improving temporal and disposition-driven efficiencies should be a focus of future research, especially in light of the multiple challenges facing smaller rural hospitals with no in-house specialty psychiatric providers and no inpatient psychiatric beds.

\section{Acknowledgments}

This study was supported by the HRSA of the U.S. Department of Health and Human Services (HHS) under grant number G01RH27871 and title Evidence-Based Teleemergency Network Grant Program. The content and conclusions of this study are those of the authors and should not be construed as the official position or policy of, nor should any endorsement be inferred by, HRSA, HHS, or the U.S. Government.

\section{Disclosure Statement}

S.L, D.H, and H.R. are employed by Union Hospital, which serves as the hub for the WVRTN Telehealth Network and provides telemedicine services. None of the other authors has competing financial interests to report.

\section{REFERENCES}

1. Broderick A, Lindeman D. Scaling telehealth programs: Lessons from early adopters. Commonwealth Fund 2013;1:1654.
2. Meyer J, McKean A, Blegen R, Demaerschalk B. Emergency Department Telepsychiatry Service Model for a Rural Regional Health System: The First Steps. Telemed J E Health 2019;25:18-24.

3. Ekeland AG, Bowes A, Flottorp S. Effectiveness of telemedicine: A systematic review of reviews. Int J Med Inform 2010;79:736-771.

4. Turvey C, Fortney J. The use of telemedicine and mobile technology to promote population health and population management for psychiatric disorders. Curr Psychiatry Rep 2017;19:88.

5. Rachal J, Sparks W, Zazzaro C, Blackwell T. Highlight in Telepsychiatry and Behavioral Health Emergencies. Psychiatr Clin North Am 2017;40:585-596.

6. Mohr N, Vakkalanka J, Ward M, et al. Telemedicine Use Decreases Rural Emergency Department Length of Stay for Transferred North Dakota Trauma Patients. Telemed J E Health 2018;24:194-202.

7. Gindi RM, Black LI, Cohen RA. Reasons for emergency room use among U.S. adults aged 18-64: National Health Interview Survey, 2013 and 2014, National health statistics reports; no 90. Hyattsville: National Center for Health Statistics, 2016.

8. Moore BJ, Stocks C, Owens PL. Trends in emergency department visits, 20062014. HCUP Statistical Brief \#227. Rockville, MD: Agency for Healthcare Research and Quality, 2017.

9. STATSAmerica. (2016). USA Counties in Profile. Available at www.statsamerica.org/uscp/default.aspx (last accessed August 7, 2018).

10. Robert Wood Johnson Foundation (2018). County Health Rankings and Roadmaps. Available at www.countyhealthrankings.org/app/indiana/2018/ overview (last accessed August 6, 2018).

11. Health Resources and Services Administration (HRSA) (2017). HRSA Data Warehouse, HPSA Find Results: State: Indiana; Discipline: Mental Health. Available at: https://datawarehouse.hrsa.gov/tools/analyzers/HpsaFindResults.aspx (last accessed July 28, 2018)

12. Mohr NM, Young T, Harland KK, Skow B, Wittrock A, Bell A, Ward MM. Emergency department telemedicine shortens rural time-to-provider and emergency department transfer times. Telemed J E Health 2018;24: 583-593.

13. Shore JH, Yellowlees $P$, Caudill $R$, Johnston B, Turvey $C$, et al. Best practices in video-conferencing-based telemental health April 2018. Telemed J E Health 2018;24;1-6.

14. Fortney JC, Pyne, JM, Kimbrell TA, Hudson TJ, Robinson DE, et al. Telemedicinebased collaborative care for post-traumatic stress disorder: A randomized clinical trial. JAMA Psychiatry 2015;72:58-67.

15. Fortney JC, Pyne, JM, Edlund MJ, Williams DK, Robinson DE, et al. A randomized trial of telemedicine-based collaborative care for depression. J Gen Intern Med 2007;22:1086-1093.

16. Fortney JC, Pyne, JM, Mouden SB, Mittal D, Hudson TJ, et al. Practice-based versus telemedicine-based collaborative care for depression in rural federally qualified health centers: A pragmatic randomized comparative effectiveness trial. Am J Psychiatry 2013;170:414-425.

17. Kocsis BJ, Yellowlees P. Telepsychotherapy and the therapeutic relationship: Principles, advantages and case examples. Telemed J E Health 2018; 24:329-334.

18. Hollander JE, Davis TM, Doarn C, Goldwater JC, Klasko S, Lowery C, Papanagnou $D$, Rasmussen P, Sites FD, Stone D, Carr BG. Recommendations from the first national academic consortium of telehealth. Pop Health Manag 2018;21;271277.

19. von Elm E, Altman DG, Egger M, Pocock SJ, Gøtzsche PC, et al. The Strengthening the Reporting of Observational Studies in Epidemiology (STROBE) Statement: Guidelines for Reporting Observational Studies. PLoS Med 2007;4:e296

20. Fairchild RM, Ferng SF, Laws S, O'Brien A, Rahmouni H. Perceptions of Rural Emergency Department Providers Regarding Telehealth-Based Care: Perceived Competency, Satisfaction with Care and Tele-ED Patient Disposition. Open J Nurs $2017 ; 7 ; 721-733$. 


\section{FAIRCHILD ET AL.}

21. Drummond MF, O'Brien B, Stoddart GL, Torrance GW. Methods for the economic evaluation of healthcare programmes, 3rd ed. Oxford, UK: Oxford University Press, 2005.

22. Bureau of Labor Statistics. Consumer Price Index (CPI values) 2005-2013). Available at www.bls.gov/cpi/ (last accessed on March 10, 2018).

23. Centers for Disease Control. Introduction to Meaningful Use, Health Information Technology for Economic and Clinical Health (HITECH) Act of Centers for Medicare and Medicaid (CMS), updated January 2017. Available at www.cdc.gov/ehrmeaningfuluse/introduction.html (last accessed on August 25, 2018).

24. Harris Y, Gilman B, Ward M, Ladinsky J, Crowley J, Warren C, Caplan C. Building the evidence for tele-emergency care: Efforts to identify a standardized set of outcome measures. Telemed J E Health 2017;23:561-566.

25. Agency for Healthcare Research Quality (AHRO). Clinical Classification software categorization guide. Available at www.hcupus.ahrq.gov/ toolssoftware/ccs/CCSCategoryNames_FullLabels.pdf (last accessed January 20, 2016).

26. McDonald J. Handbook of Biological Statistics, 3rd ed. Baltimore, MD: Sparky House Publishing, 2014.

27. Manikandan S. Data transformation. J Pharmacol Pharmacother 2010;1:126127.

28. Interdepartmental Serious Mental IIIness Coordinating Committee (ISMICC). (2017). The Way Forward: Federal Action for a System That Works for All People Living With Serious Mental IIIness (SMI) and Serious Emotional Disturbance
(SED) and Their Families and Caregivers. Department of Health and Human Services. Available at www.samhsa.gov/about-us/advisory-councils/ismicc (last accessed October 3, 2018).

29. Yilmaz $S$, Horn B, Fore $C$, Bonham C. An economic cost analysis of an expanding, multi-state behavioural telehealth intervention. J Telemed Telecare 2018 [Epub ahead of print]; DOI: 10.1177/1357633X18774181.

Address correspondence to: Roseanne Moody Fairchild, PhD, RN, CNE, NE-BC Nursing and Health Professions State College of Florida, Manatee-Sarasota 7131 Professional Parkway East Bradenton, FL 34240

E-mail: fairchr@scf.edu

Received: August 29, 2018

Revised: November 16, 2018

Accepted: November 21, 2018

Online Publication Date: February 8, 2019 